\title{
A multiwavelength study of the remnant of nova GK Persei
}

\author{
G. C. Anupama ${ }^{1}$ and N. G. Kantharia ${ }^{2}$ \\ ${ }^{1}$ Indian Institute of Astrophysics, II Block Koramangala, Bangalore 560 034, India \\ e-mail: gca@iiap.res.in \\ ${ }^{2}$ National Centre for Radio Astrophysics, Tata Institute of Fundamental Research, Post Bag 3, Ganeshkhind, \\ Pune 411 007, India \\ e-mail:ngk@ncra.tifr.res.in
}

Received 16 November 2004 / Accepted 20 January 2005

\begin{abstract}
We present new observations of the nebular remnant of the old nova GK Persei 1901 in the optical using the Himalayan Chandra Telescope (HCT) and at low radio frequencies using the Giant Metrewave Radio Telescope (GMRT). The dimensions of the shell in the optical emission lines of [NII], [OIII] and [OII] are $108 \times 94 \operatorname{arcsec}^{2}, 104 \times 94 \operatorname{arcsec}^{2}$ and $99 \times 87 \mathrm{arcsec}^{2}$, respectively. The evolution of the nova remnant indicates shock interaction with the ambient medium, especially in the southwest quadrant. Application of a simple model for the shock and its evolution to determine the time dependence of the radius of the shell in the southwest quadrant indicates that the shell is now expanding into an ambient medium that has a density of $0.12 \mathrm{~cm}^{-3}$, compared to the density of the ambient medium of $0.8 \mathrm{~cm}^{-3}$ ahead of the shock in 1987 . There are indications of a recent interaction of the nova remnant with the ambient medium in the northeast quadrant. There is a distinct flattening of the shell, as well as an increase in the number and brightness of the knots in the region. The brightest optical knots in this region are also detected in the radio images.

The nova remnant of GK Per is detected at all the observed radio frequencies and is of a similar extent to the optical remnant. Putting together our radio observations with VLA archival data on GK Per from 1997, we obtain three interesting results: 1. the spectrum above $1.4 \mathrm{GHz}$ follows a power law with an index $-0.7\left(S \propto v^{\alpha}\right)$ and below $1.4 \mathrm{GHz}$ follows a power law with an index $\sim-0.85$. This could be due to the presence of at least two populations of electrons dominating the global emission at different frequencies; 2 . we record an annual secular decrease of $2.1 \%$ in the flux density of the nova remnant at $1.4 \mathrm{and} 4.9 \mathrm{GHz}$ between 1984 and 1997 which has left the spectral index unchanged at -0.7 . No such decrease is observed in the flux densities below $1 \mathrm{GHz} ; 3$. we record an increase in the flux density at $0.33 \mathrm{GHz}$ compared to the previous estimate in 1987 . We conclude that the remnant of nova GK Per is similar to supernova remnants and in particular to the young supernova remnant Cas A.
\end{abstract}

Key words. novae, cataclysmic variables - ISM: general

\section{Introduction}

Cataclysmic variables (CVs) are interacting binary systems with a white dwarf primary accreting material from its Rochelobe filling main-sequence companion (Warner 1995). These systems have short orbital periods of the order of a few hours. Classical novae are a subset of CVs which undergo outbursts on the surface of the white dwarf due to thermonuclear runaway in the accretion material. The outbursts result in the ejection of $\sim 10^{-4} M_{\odot}$ of material at velocities of up to several thousand kilometers per second. GK Persei is a classical nova system that had an outburst in 1901, and is unique in many respects. It is now classified as a fast "neon" nova. The nova binary consists of a magnetic white dwarf with a surface magnetic field of $5 \times 10^{5} \mathrm{G}$ (Bianchini \& Sabbadin 1983) and an evolved latetype (K2IV) companion. The orbital period of 1.904 days is the longest known for a classical nova system. In contrast with most old novae, this system exhibits dwarf nova-like outbursts of $\sim 3 \mathrm{mag}$.
A month after the 1901 outburst, rapidly expanding nebulosities were detected in the vicinity of the nova (e.g. Ritchey 1901; Perrine 1902). This first recorded "light echo" was explained in terms of reflection from dust grains lying in a plane crossing the line of sight to the nova (Couderc 1939). The expanding ejecta from the nova outburst was first discovered in 1916 and has been observed intermittently since then in the broad bands as well as in emission lines. Seaquist et al. (1989, hereinafter S89) present a detailed study of the evolution of the optical nebulosity until 1988. The sequence of the optical images indicate that the ejecta are being decelerated, particularly in the southwest quadrant, by interaction with the ambient medium. The optical evolution of the shell later has been studied by Anupama \& Prabhu (1993), Slavin et al. (1995) and Lawrence et al. (1995).

The confirmation of the strong interaction in the southwest quadrant comes from the detection of a ridge of non-thermal, synchrotron emission in the radio, coincident with the flattening in the optical images in the southwest quadrant (S89). 
ROSAT HRI images and the CHANDRA images in the X-rays (Balman \& Ögelman 1999; Balman 2002) indicate that the nebula is brightest in the southwest quadrant and towards the west with a lumpy morphology and that it resembles the radio shell. The X-ray shell is predicted to be in a transition to the Sedov phase. The nova ejecta of GK Per resemble a young supernova remnant.

A search for the ambient medium (Bode et al. 1987; S89) resulted in the detection of an extended emission in the farinfrared. This extended emission runs roughly northwest to southeast through the position of the nova and has a total extent of 30 arcmin. It is double peaked with the nova lying on a saddle point between the peaks. A similar extended emission is seen in HI and CO emission (S89; Scott et al. 1994). A re-analysis of the high resolution IRAS images by Dougherty et al. (1996) indicates that the extended far infrared emission is due to dust at $T=23 \pm 1 \mathrm{~K}$ with $M_{\mathrm{d}}=0.04 M_{\odot}$, while the $\mathrm{HI}$ mass in the same region is $1 M_{\odot}$.

A faint optical bipolar nebula extending well beyond the current nova remnant was first detected by Tweedy (1995) and subsequently by Bode et al. (2004). A detailed study of the images of the nebulosity in $\mathrm{H} \alpha$ and [OIII] emission by Bode et al. (2004) indicate that it is correlated with the light echoes detected during the 1901 outburst. The brightest region of this large-scale optical nebulosity is also coincident with the longest lived of the light echoes of 1902. The encounter of the expanding nova ejecta with the high density regions of this bipolar nebula leads to shocks and particle acceleration that are seen as radio and X-ray emission, and are seen also in the clumpy nature and deceleration of the nova remnant in the optical.

Bode et al. (1987) first suggested that the IRAS emission might be due to material ejected in a previous phase of the evolution of the central binary and could be a fossil planetary nebula. Dougherty et al. (1996) and Bode et al. (2004) later suggested the origin of the material to be a "born again" AGB phase of the binary as the white dwarf accreted material at a very high rate from the secondary star, creating a common envelope which was then ejected. This evolutionary model is consistent with the current mass of the secondary which is low for its luminosity, the $\sim 1 M_{\odot}$ material present in the fossil material and the detection of the extended bipolar optical nebula. An estimate of the proper motion of the central system together with the radial velocity of the system indicates a space velocity of $45 \pm 4 \mathrm{~km} \mathrm{~s}^{-1}$ (Bode et al. 2004).

We present in this paper a study of the nebular remnant in low-frequency radio emission as well as in the optical. The evolution of the nova remnant since the detailed work by S89 is presented. We also present a study of the environment in HI. We follow Mclaughlin (1960) in assuming that GK Per is located at a distance of $470 \mathrm{pc}$. At this distance, $1^{\prime}$ corresponds to $0.14 \mathrm{pc}$.

\section{Observations and data analysis}

\subsection{Optical}

Optical CCD images of the nebular shell around GK Per were obtained through Bessell $U B V R, \mathrm{H} \alpha+[\mathrm{NII}]$ (100 ̊
Table 1. Optical observations.

\begin{tabular}{lcc}
\hline \hline $\begin{array}{l}\text { Date } \\
(2003)\end{array}$ & Filter & $\begin{array}{c}\text { Exposure time } \\
(\mathrm{s})\end{array}$ \\
\hline January 2 & $U$ & 2400 \\
& $B$ & 540 \\
& $V$ & 180 \\
& $R$ & 120 \\
& $I$ & 180 \\
& $\mathrm{H} \alpha+[\mathrm{N} \mathrm{II}]$ & 2400 \\
November 24 & {$[\mathrm{O} \mathrm{III]}]$} & 3900 \\
& $U$ & 4200 \\
& $B$ & 240 \\
& $V$ & 180 \\
& $R$ & 65 \\
& $I$ & 20 \\
& $\mathrm{H} \alpha+[\mathrm{N} \mathrm{III}]$ & 1800 \\
& {$[\mathrm{O} \mathrm{III]}]$} & 4200 \\
\hline
\end{tabular}

bandpass) and [OIII] (100 ̊̊ bandpass) filters on 2 January and 24 November 2003 using the HFOSC instrument on the 2-m Himalayan Chandra Telescope (HCT). The total field of view is $10^{\prime} \times 10^{\prime}$ with an image scale of $0.297 \mathrm{arcsec} / \mathrm{pixel}$. More details on the telescope and the instrument may be obtained at http://www.iiap.ernet.in/ iao.

The details of the observations are given in Table 1 . The seeing was poor on both occasions, and ranged between $2^{\prime \prime}-2.5^{\prime \prime}$. All images were bias subtracted and flat-field corrected in the standard manner using the various tasks in the IRAF package.

\subsection{Radio}

GK Per was observed in the radio continuum at $0.33 \mathrm{GHz}$, $0.61 \mathrm{GHz}$ and $1.28 \mathrm{GHz}$ and in $21 \mathrm{~cm} \mathrm{HI}$ using the Giant Metrewave Radio Telescope (GMRT). All observations, except in the $1.28 \mathrm{GHz}$, were made from August-October 2002. The $1.28 \mathrm{GHz}$ radio continuum observations were made in October 2003. The observations started and ended with a run on an amplitude calibrator (3C 147, 3C 286) which also doubled as bandpass calibrators for the radio continuum observations. 3C 287 was used as the bandpass calibrator for the $21 \mathrm{~cm} \mathrm{HI}$ observations. The on-source runs were interspersed with short runs on a phase calibrator $(0432+416)$. We obtained an average of 5-6 $\mathrm{h}$ on-source data for all the bands. The observational details are summarized in Table 2.

The data were converted to standard FITS format and imported to NRAO AIPS. The GMRT always works in the spectral line mode giving 128 channels for each sideband and each polarization. Hence, the GMRT data have to be first gain calibrated, then bandpass calibrated and then the channels averaged to obtain the continuum database. Thus the analysis strategy in AIPS, generally followed for GMRT continuum data, is that a single channel data on the amplitude and bandpass calibrators is initially examined for bad data, edited and then gain calibrated. The bandpass calibrator is then used to generate the bandpass gain solutions. The bandpass calibration is then applied to all the data and line-free/RFI-free channels averaged 
Table 2. Details of the radio observations.

\begin{tabular}{lcccc}
\hline \hline Detail & $0.33 \mathrm{GHz}$ & $0.61 \mathrm{GHz}$ & $1.28 \mathrm{GHz}$ & $21 \mathrm{~cm}$ \\
\hline Date of observation & $20 / 8 / 2002$ & $5 / 9 / 2002$ & $16 / 10 / 2003$ & $25 / 9 / 2002$ \\
Baseband bandwidth (MHz) & 16 & 16 & 16 & 1 \\
Flux density of phase cal 0432+416 (Jy) & $17.15 \pm 0.08$ & $14.3 \pm 0.18$ & $10.9 \pm 0.13$ & $10.5 \pm 1.2$ \\
Primary beam size & $108^{\prime}$ & $54^{\prime}$ & $26^{\prime}$ & $24^{\prime}$ \\
Synthesized beam $^{1}$ & $13^{\prime \prime} \times 11^{\prime \prime}$ & $14.4^{\prime \prime} \times 11^{\prime \prime}$ & $25^{\prime \prime} \times 12.5^{\prime \prime}$ & $37^{\prime \prime} \times 28^{\prime \prime}$ \\
Continuum/line rms (mJy/beam) & 0.7 & 0.35 & 0.14 & 2 \\
\hline
\end{tabular}

${ }^{1}$ This is the synthesized beam of the images presented in the paper and, in most cases, is larger than the best achievable.

to generate a continuum uv data base. The continuum data on the phase calibrator are then used to gain calibrate the target field. The calibrated target field is then imaged. All the fields have been corrected for the primary beam gain variation.

Since wide-field effects start dominating at the lower frequencies, we imaged the $0.33 \mathrm{GHz}$ primary beam using 25 facets and the $0.61 \mathrm{GHz}$ primary beam using 9 facets. The $1.28 \mathrm{GHz}$ and $21 \mathrm{~cm}$ data were imaged using simple 2-D FFT algorithms in AIPS.

The HI $21 \mathrm{~cm}$ line data was treated slightly differently than the radio continuum bands. The data was bandpass calibrated using 3C 287. A data cube was then generated from this calibrated database on GK Per by using a uv-taper of $20 \mathrm{k} \lambda$. The channel separation was $1.6 \mathrm{~km} \mathrm{~s}^{-1}$ and the rms noise on the channel images was $2.3 \mathrm{mJy} / \mathrm{beam}$.

We have also analysed the $1.4 \mathrm{GHz}$ and $4.9 \mathrm{GHz}$ VLA data on GK Per obtained in 1997 and available in the NRAO Data Archives.

\section{Results}

\subsection{Optical images}

The nebular remnant of the 1901 nova outburst is clearly detected in the $\mathrm{H} \alpha+[\mathrm{NII}]$ and [OIII] images. The shell is also clearly seen in the $U$ band images, and could possibly be due to [OII] $3727 \AA$ emission.

Figure 1a shows the remnant in the $\mathrm{H} \alpha+[\mathrm{NII}]$. The images of the remnant presented by Slavin et al. (1995) and Lawrence et al. (1995) clearly indicate that the $\mathrm{H} \alpha+[\mathrm{NII}]$ image presented here is predominantly due to emission from [NII]. The shell is boxy and asymmetric, with a flattening in the northeast and southwest quadrants (see Fig. 1). The major axis of the shell lies approximately southeast to northwest, with a major to minor axis ratio of 1.15 . The shell is inhomogeneous and consists of blobs of emission of varying size and surface brightness, with the bulk of the emission arising from the southern region. The remnant appears to be three quarters of a rectangle, with a depletion of emission knots in the eastern edge. The shell extends to only 45 arcsec to the east, while it extends to 53 arcsec to the west. The shell appears more symmetric along the north-south direction, with the radius of the shell being approximately 49 arcsec in both directions. The dimension of the shell along the major and minor axes is $108 \times 94 \mathrm{arcsec}^{2}$, while it was $103 \times 90 \operatorname{arcsec}^{2}$ in 1993 (Slavin et al. 1995).
Figure $1 \mathrm{~b}$ shows the shell in [OIII] emission. The general morphology of the shell is similar to [NII]. The flattening in the northeast quadrant is also present in the [OIII] image. The dimension of the shell is $104 \times 94 \operatorname{arcsec}^{2}$, with a major axis to minor axis ratio of 1.1 . In contrast to the [NII] emission, the emission knots are more evenly distributed in [OIII]. The eastern hole is also not very prominent. On the other hand the shell is more extended to the east compared to its extent in the west.

Figure 1c shows the shell in [OII] emission. The general morphology is very similar to the [NII] emission. The dimension of the shell is $99 \times 87 \operatorname{arcsec}^{2}$ and the axes ratio is 1.14 , similar to the [NII] shell. The emission "hole" in the east is very prominent, and as in the case of [NII], the bulk of the emission is in the south-southwest region.

Faint optical nebulosity extending well beyond the current nova ejecta was first detected by Tweedy (1995) in both $\mathrm{H} \alpha$ and [OIII] emission. Bode et al. (2004) have also detected this nebulosity in both emissions. Our $\mathrm{H} \alpha+[\mathrm{NII}]$ and [OIII] images also show this extended nebulosity. The nebulosity in $\mathrm{H} \alpha$ is shown in Fig. 1d.

The nebulosity has an "hourglass" shape that is slightly tilted, and also flattened towards the southwest. The brightest regions are the flattened portion to the southwest, and a "jet-like feature" to the northeast. The [OIII] emission lies inwards of the $\mathrm{H} \alpha$ emission, except for the "jet-feature" that is coincident in both emissions. Comparing the data to the IRAS $100 \mu \mathrm{m}$ images and the light echo images seen in 1902, Bode et al. (2004) find that the southwest flattening in the [OIII] coincides with the infrared emission, while in the $\mathrm{H} \alpha$, it coincides with the longest-lived of the light echoes of 1902. Bode et al. (2004) suggest that the flattening is caused by a deceleration of the nebula due to interaction with the interstellar medium in the direction of the motion of the system.

\subsection{Radio}

The naturally weighted images of the nova remnant at $0.33 \mathrm{GHz}, 0.61 \mathrm{GHz}$ and $1.28 \mathrm{GHz}$ are shown in Fig. 2. A uv-range of $20 \mathrm{k} \lambda$ was used at $1.28 \mathrm{GHz}$ so as to match the $0.33 \mathrm{GHz}$ uv-cutoff. While the grey scale in the figure has been plotted down to $2 \sigma$, the contours start from $3 \sigma$. The southwest ridge of emission is seen at all frequencies. While the locations of the peak emission at the $0.33 \mathrm{GHz}$ and $0.61 \mathrm{GHz}$ are not 


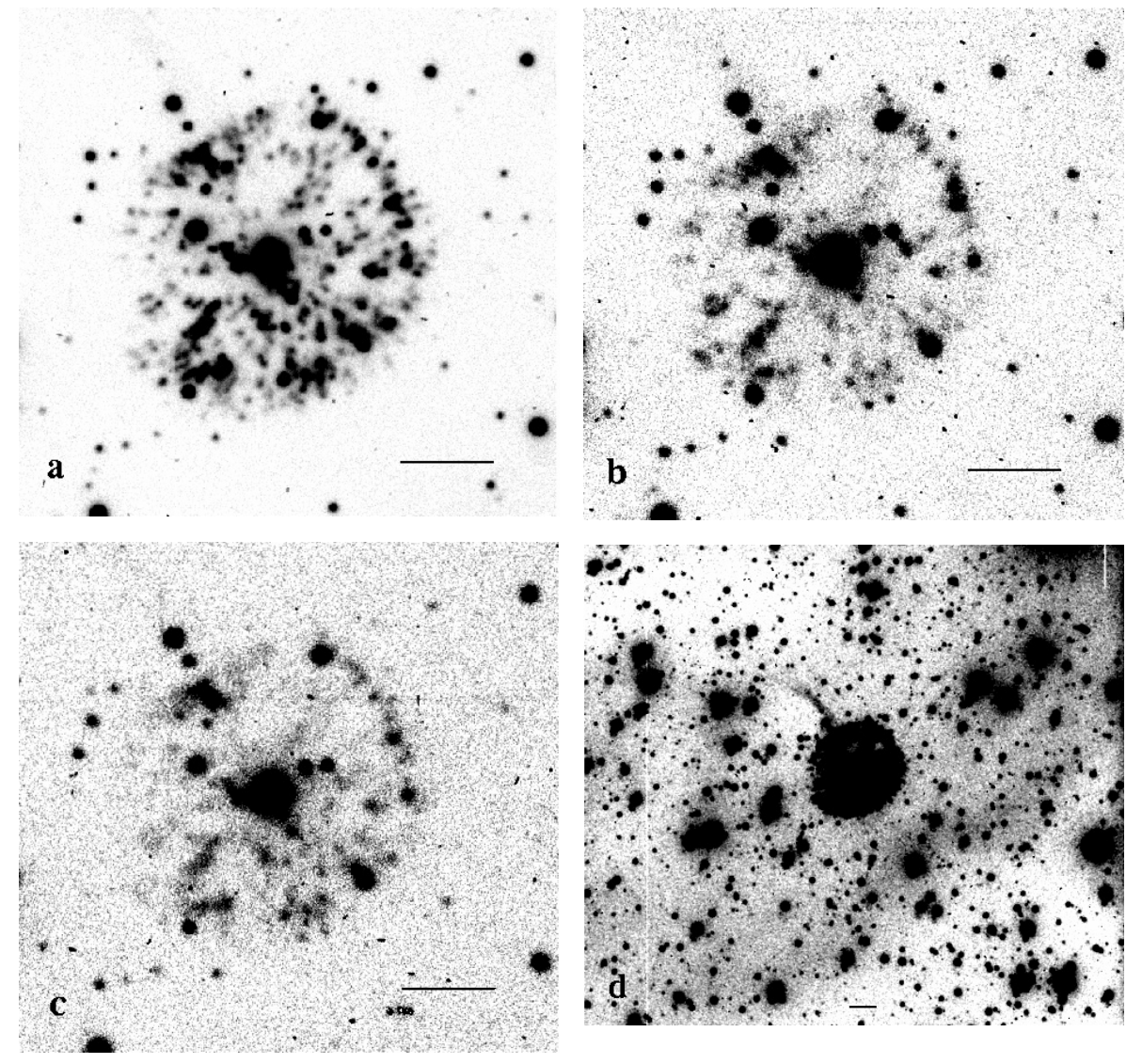

Fig. 1. The nova remnant in a) $\mathrm{H} \alpha+[\mathrm{N} \mathrm{II}]$; b) $\left[\mathrm{O} \mathrm{III]}\right.$; and c) [O II]. All images are $2.9^{\prime} \times 3.2^{\prime}$; d) the extended bipolar nebula in $\mathrm{H} \alpha$. Note the "jet" feature in the northeast quadrant. The image is $10^{\prime} \times 10^{\prime}$. All images are with north up and east to the left. The scale bar is $30^{\prime \prime}$ in length.
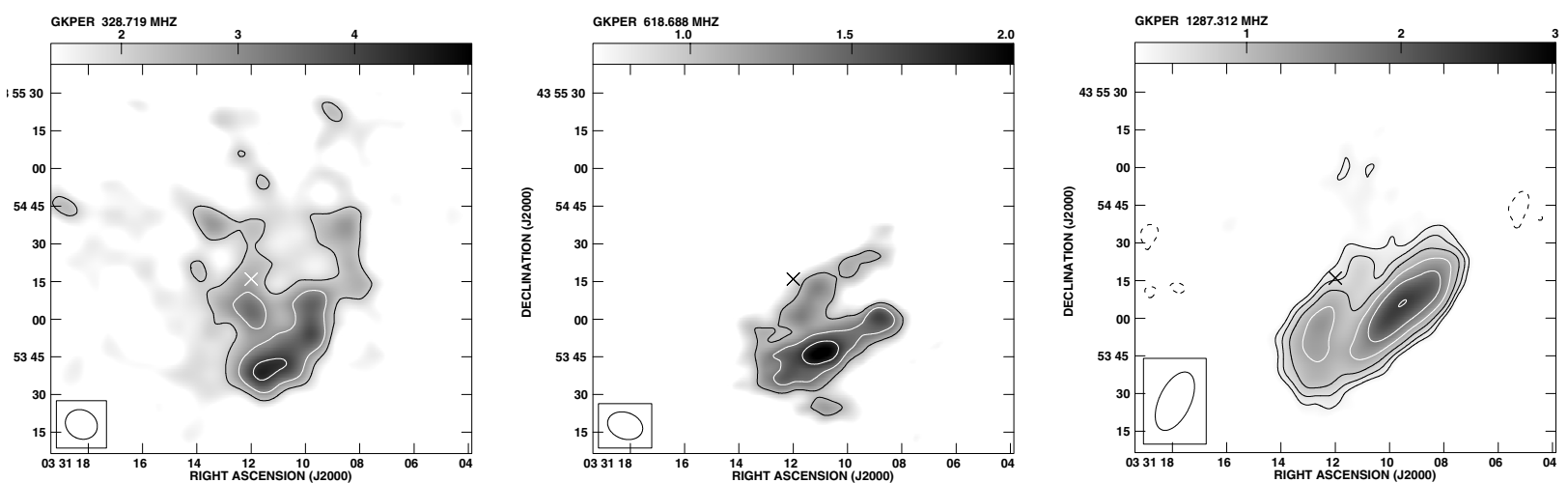

Fig. 2. GMRT naturally weighted $0.33 \mathrm{GHz}$ (left), $0.61 \mathrm{GHz}$ (center) and $1.28 \mathrm{GHz}$ (right) images of the radio emission from the GK Persei remnant. The angular resolution is $13^{\prime \prime} \times 11^{\prime \prime}$ at a PA $=61.2$ and the rms noise on the $0.33 \mathrm{GHz}$ image is $0.7 \mathrm{mJy} / \mathrm{beam}$. The grey scale ranges from $1.4(2 \sigma)$ to $5 \mathrm{mJy} /$ beam. The angular resolution is $21.9^{\prime \prime} \times 13.0^{\prime \prime}$ at a $\mathrm{PA}=69.9^{\circ}$ and the rms noise on the $0.61 \mathrm{GHz}$ image is $0.35 \mathrm{mJy} /$ beam. The grey scale ranges from $(2 \sigma)$ to $2 \mathrm{mJy} /$ beam. The angular resolution is $25.04^{\prime \prime} \times 12.52^{\prime \prime}$ at a PA $=-25.5^{\circ}$ and the rms noise on the $1.28 \mathrm{GHz}$ image is $0.14 \mathrm{mJy} / \mathrm{beam}$. The grey scale ranges from $2 \sigma$ to $3 \mathrm{mJy} / \mathrm{beam}$. In all images, the contours are plotted starting from $3 \sigma$ and increased in steps of $\sqrt{(2)}$. Note that we do not detect the extended low brightness emission in the northeast at $0.61 \mathrm{GHz}$ and $1.28 \mathrm{GHz}$. The cross marks the position of the nova.

too different, the location of the peak emission at $1.28 \mathrm{GHz}$ is quite different. Moreover, the $0.61 \mathrm{GHz}$ image shows features evident in the images at the other two frequencies.

There are three strong sources (we refer to these as source A, B following S89 and source C) which appear in the primary beam (see Table 2) at all the observed frequencies and limit the dynamic range of our images.
The flux density of the remnant of GK Per estimated at the three radio frequencies and the spectral index implied by these are listed in Table 3. The spectral index between 0.33 and $0.61 \mathrm{GHz}$ varies from -0.4 to -1.6 across GK Per. The spectral index is steepest along a ridge running from north to southwest. The global spectral index of the remnant between $0.33 \mathrm{GHz}$ and $1.28 \mathrm{GHz}$ is -0.85 . Unlike $\mathrm{S} 89$, whose data 
Table 3. Flux density of GK Per at the three observed frequencies. The spectral index is calculated for the frequency in the same row and the next frequency. The spectral index $\alpha\left(S_{v} \propto v^{\alpha}\right)$ in the first row is between 0.33 and $0.61 \mathrm{GHz}$ and in the third row is between 0.33 and $1.28 \mathrm{GHz}$.

\begin{tabular}{cccc}
\hline $\begin{array}{c}\text { Frequency } \\
\mathrm{GHz}\end{array}$ & $\begin{array}{c}\text { Flux density } \\
\mathrm{mJy}\end{array}$ & Spectral index $\alpha$ & $\begin{array}{c}\text { S89 } \\
\mathrm{mJy}\end{array}$ \\
\hline 0.33 & $36.8 \pm 4$ & $-0.85_{-0.15}^{+0.2}$ & $20_{-5}^{+10}$ \\
0.61 & $21.8 \pm 0.5$ & $-0.87_{-0.11}^{+0.08}$ & $23 \pm 3$ \\
1.28 & $11.4 \pm 0.5$ & $-0.86( \pm 0.11)$ & - \\
\hline
\end{tabular}

suggested that the radio spectrum of GK Per turned over around $1 \mathrm{GHz}$, we find that the spectrum is clearly a power law down to $0.33 \mathrm{GHz}$. S89 estimated a spectral index of -0.7 between $1.4 \mathrm{GHz}$ and $4.9 \mathrm{GHz}$. Analysis of the VLA archival data from 1997 at $1.4 \mathrm{GHz}$ and $4.9 \mathrm{GHz}$ gives a similar spectral index. It thus appears that the spectral index above $1.4 \mathrm{GHz}$ is -0.7 and below $1.4 \mathrm{GHz}$ is -0.85 . There does appear to be a spectral break near $1 \mathrm{GHz}$ but the spectrum becomes steeper at lower frequencies.

\subsection{1. $21 \mathrm{~cm} \mathrm{HI}$}

$21 \mathrm{~cm} \mathrm{HI} \mathrm{emission} \mathrm{at} 4.9 \mathrm{~km} \mathrm{~s}^{-1}$ was detected in a $\sim 15^{\prime}$ strip running northwest to southeast with a gap in the central parts near the nova position. This corresponds to a linear extent of about 2.1 pc. This has been earlier reported by S 89 who also found it to coincide with the IRAS FIR emission from dust. A spectrum integrated over a small region in the north of this emission is shown in Fig. 3. The emission extends from about $20 \mathrm{~km} \mathrm{~s}^{-1}$ to $-25 \mathrm{~km} \mathrm{~s}^{-1}$ and is strongest near $4.9 \mathrm{~km} \mathrm{~s}^{-1}$, which is similar to what $\mathrm{S} 89$ found. The HI emission extends on both sides of the nova and is along the ridge of radio continuum emission from the nova remnant, indicating that the HI emission is related to the nova. The $\mathrm{HI}$ emitting region is large and there is considerable flux missing at low spatial frequencies due to missing short spacings in the interferometer data. Thus we do not estimate the column density and mass of HI from this data. We also detect $\mathrm{HI}$ emission from a region to the west of GK Per near $-5 \mathrm{~km} \mathrm{~s}^{-1}$.

To further examine the neutral gas near GK Per, we obtained the absorption spectra towards the three strong sources seen in the primary beam. The flux densities of these sources at the three observed frequencies and the HI optical depth in these directions are listed in Table 4 . The flux density of source B at $1.4 \mathrm{GHz}$ is uncertain since it lies close to the edge of the primary beam. The flux density of these objects that we estimate at $1.28 \mathrm{GHz}$ and $1.4 \mathrm{GHz}$ differs from those noted in $\mathrm{S} 89$ by a significant amount. To resolve the issue, we compared our flux densities with NVSS and find a good correlation as noted in Table 4. We, therefore, believe that the values quoted by S89 are in error, probably because they did not correct for the primary beam gain variation. We detect the largest optical depth $(\sim 0.35)$ at a velocity of $-1.5 \mathrm{~km} \mathrm{~s}^{-1}$ towards source $\mathrm{C}$, which is about $3^{\prime}$ north of GK Per. The absorption spectrum towards this source is shown in Fig. 4. This extragalactic source, which is projected onto the extended HI nebula, is not mentioned by

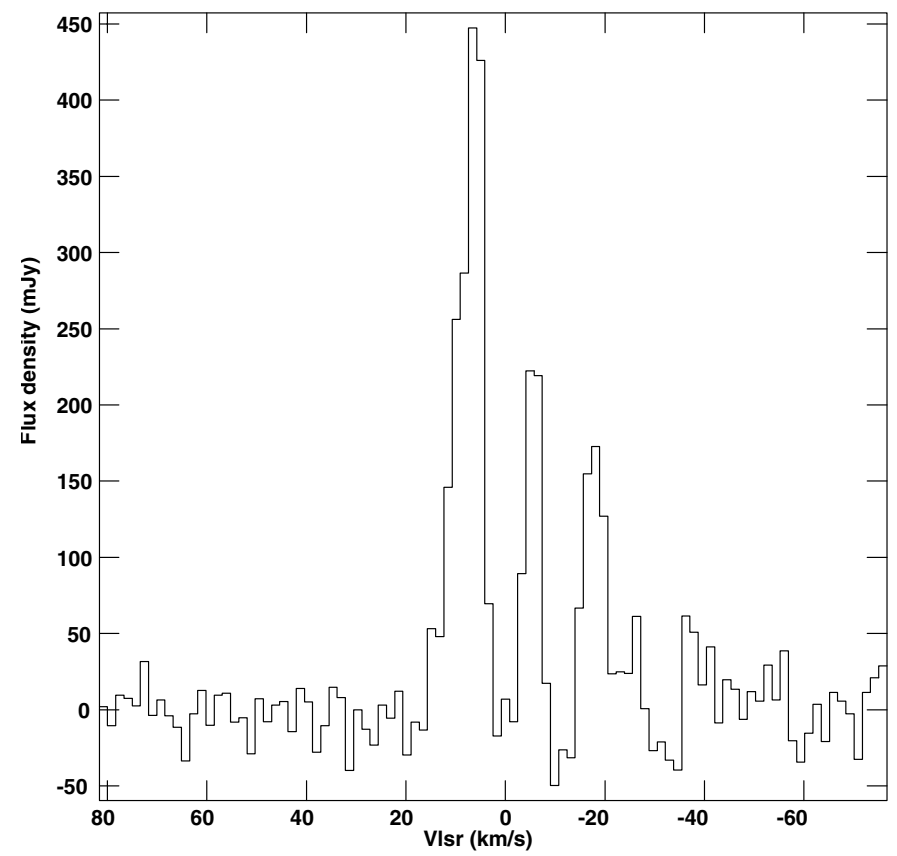

Fig. 3. $21 \mathrm{~cm} \mathrm{HI} \mathrm{emission} \mathrm{integrated} \mathrm{over} \mathrm{a} \mathrm{small} \mathrm{region} \mathrm{in} \mathrm{the} \mathrm{north-}$ west of the nova GK Per.

S89. Moreover, we do not detect any absorption near $4.9 \mathrm{~km} \mathrm{~s}^{-1}$ in front of source $\mathrm{C}$, although the emission observed at this velocity seems to extend to the source. This could be due to the absence of detectable absorbing gas in front of the source at this velocity. Using the column density of $5.4 \times 10^{20} \mathrm{~cm}^{-2}$, for the $5 \mathrm{~km} \mathrm{~s}^{-1}$ and $0 \mathrm{~km} \mathrm{~s}^{-1}$ features (S89), and for a limiting optical depth of 0.2 , we estimate that the spin temperature of the gas near $5 \mathrm{~km} \mathrm{~s}^{-1}$ should be $>150 \mathrm{~K}$ since we do not detect it in absorption against source $\mathrm{C}$. We do not detect significant $\mathrm{HI}$ emission centred near $-1.6 \mathrm{~km} \mathrm{~s}^{-1}$ where we detect absorption towards Source C. The spectral index of the background sources are also noted in Table 4 for completion. Source A is a steep spectrum source with a spectral index of $\sim-0.8$, source B turns over at low frequencies, while source $\mathrm{C}$ shows a flat spectrum below $0.61 \mathrm{GHz}$.

The local standard of rest velocities of the HI that we detect in absorption towards sources A and B are different from what S89 have reported (see Table 4). The velocities for objects $A$ and $B$ vary by 7.4 and $5.4 \mathrm{~km} \mathrm{~s}^{-1}$ respectively. We think that these could be due to a systematic difference of about 5$6 \mathrm{~km} \mathrm{~s}^{-1}$ between our results and S89 results. Note that the S89 data had a spectral resolution of $5 \mathrm{~km} \mathrm{~s}^{-1}$, while our data have a resolution of $1.6 \mathrm{~km} \mathrm{~s}^{-1}$. In addition, although like $\mathrm{S} 89$, we find that the extended bipolar nebula emits significantly near $5 \mathrm{~km} \mathrm{~s}^{-1}$, unlike $\mathrm{S} 89$, we do not detect much emission near $0 \mathrm{~km} \mathrm{~s}^{-1}$, which could again be due to the systematic velocity difference.

\section{Discussion}

\subsection{Evolution of the optical remnant}

The evolution of the nova shell in the optical from 1917 to 1984 is presented by S89. Anupama \& Prabhu (1993) present the 
Table 4. HI absorption and flux densities of the sources A, B and C in the GK Per field. The spectral indices between two pairs of frequencies are also listed.

\begin{tabular}{|c|c|c|c|c|c|c|c|c|c|c|c|c|c|c|}
\hline & \multirow{3}{*}{$\begin{array}{c}\text { RA (2000) } \\
\text { h m s }\end{array}$} & \multirow{3}{*}{$\begin{array}{c}\operatorname{Dec}(2000) \\
{ }^{\prime},{ }^{\prime}\end{array}$} & \multicolumn{8}{|c|}{ Radio continuum } & \multicolumn{4}{|c|}{$\overline{\mathrm{HI}}$} \\
\hline & & & \multirow{2}{*}{$\begin{array}{l}\mathrm{S}_{330} \\
\mathrm{mJy}\end{array}$} & \multirow{2}{*}{$\begin{array}{l}\mathrm{S}_{610} \\
\mathrm{mJy}\end{array}$} & \multirow{2}{*}{$\begin{array}{l}\mathrm{S}_{1280} \\
\mathrm{mJy}\end{array}$} & \multirow{2}{*}{$\begin{array}{l}S_{1420} \\
\text { mJy }\end{array}$} & \multirow{2}{*}{$\alpha_{610}^{330}$} & \multirow[t]{2}{*}{$\alpha_{1420}^{610}$} & \multirow{2}{*}{$\begin{array}{c}\text { S89 } \\
S_{1490} \\
\text { mJy }\end{array}$} & \multirow{2}{*}{$\begin{array}{c}\text { NVSS } \\
\mathrm{S}_{1420} \\
\text { mJy }\end{array}$} & \multicolumn{2}{|c|}{ GMRT } & \multicolumn{2}{|c|}{ S89 } \\
\hline & & & & & & & & & & & $\tau$ & $\begin{array}{r}\begin{array}{r}V_{\mathrm{lsr}} \\
\mathrm{km} \mathrm{s}^{-1}\end{array}\end{array}$ & $\tau$ & $\begin{array}{c}V_{\mathrm{lsr}} \\
\mathrm{km} \mathrm{s}^{-1}\end{array}$ \\
\hline A & 033032.2 & +434002 & $\begin{array}{l}614 \\
(4)\end{array}$ & $\begin{array}{l}369 \\
(2)\end{array}$ & $\begin{array}{l}192.7 \\
(0.3)\end{array}$ & $\begin{array}{l}177 \\
(3)\end{array}$ & -0.8 & -0.9 & 81.1 & 179 & 0.24 & 3.0 & 0.23 & -4.4 \\
\hline $\mathrm{B}^{*}$ & 033142.6 & +441310 & $\begin{array}{l}848 \\
(2)\end{array}$ & $\begin{array}{c}689 \\
(2)\end{array}$ & $\begin{array}{l}249.5 \\
(0.14)\end{array}$ & $\begin{array}{l}237 \\
(1)\end{array}$ & -0.3 & -1.3 & 85.3 & 335 & 0.24 & 1.9 & 0.34 & -3.5 \\
\hline $\mathrm{C}$ & 033112.4 & +435651 & $\begin{array}{l}47 \\
(2)\end{array}$ & $\begin{array}{c}47 \\
(0.7)\end{array}$ & $\begin{array}{c}33.3 \\
(0.14)\end{array}$ & $\begin{array}{c}25.8 \\
(1)\end{array}$ & 0 & -0.7 & - & 26 & 0.35 & -1.5 & - & - \\
\hline
\end{tabular}

* Source B lies close to the half power points of the GMRT $21 \mathrm{~cm}$ primary beam and hence the values based on this might be in error.

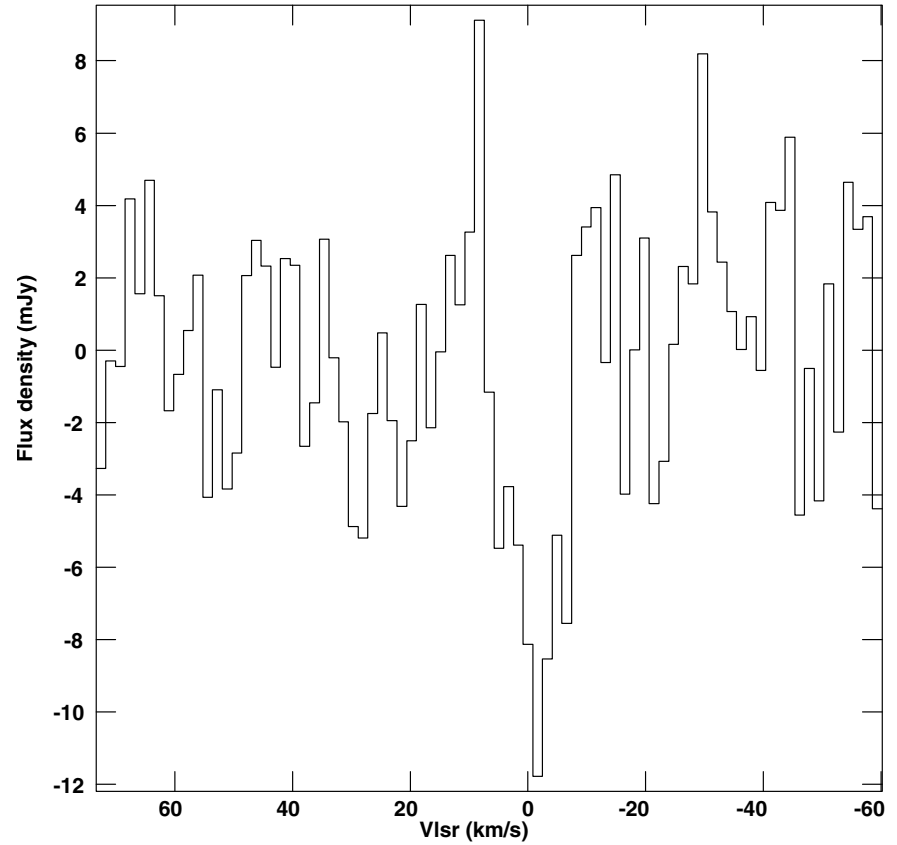

Fig. 4. $21 \mathrm{~cm} \mathrm{HI}$ absorption towards Source C located $\sim 3^{\prime}$ north of GK Per.

images of the shell obtained in 1990, while Slavin et al. (1995) present observations made in 1993. Based on a detailed study of the evolution of the remnant in the optical and its properties in the radio, S89 interpret the shell as interacting with its environment, and the flattening to the south-west is due to deceleration caused by a shock interaction of the ejecta with its ambient medium and conclude that in many respects the nova remnant of GK Per behaves like a young supernova remnant. A similar explanation is provided by Bode (2004).

S89 also provide a simple model for the shock and its evolution and determine the time dependence of the radius of the shell in the southwest quadrant. Based on both the energy conserving and momentum conserving models for the shock interaction between the nova shell and the ambient medium, they estimate the density of the medium ahead of the shock to be $0.8 \mathrm{~cm}^{-3}$.

Figure 5 shows the evolution of the radius of the southwest quadrant with time for the period 1901-2003. The radii

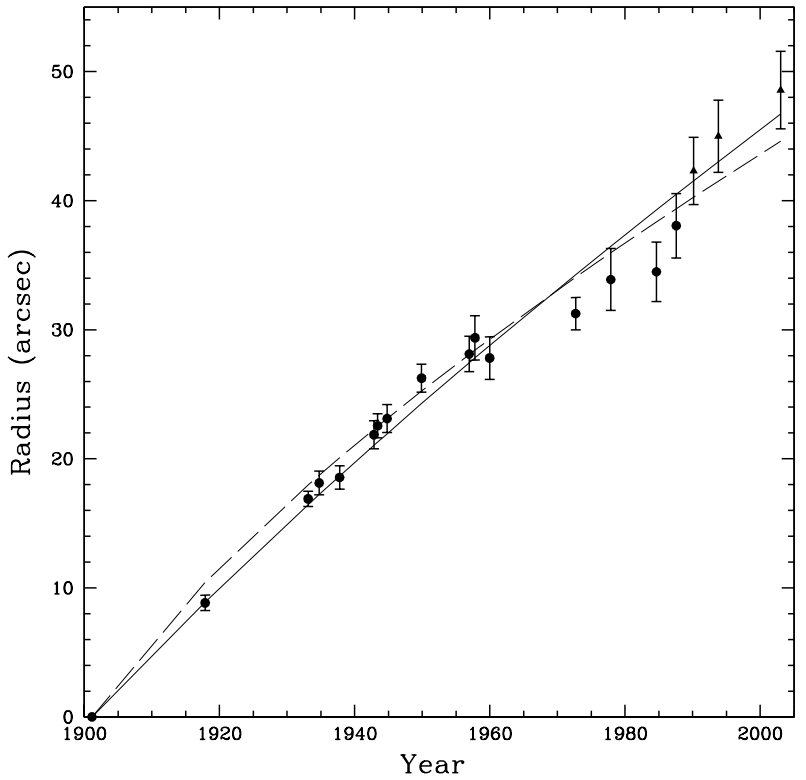

Fig. 5. Time evolution of the radius of the shell in the southwest quadrant. Filled circles refer to the radius during 1901-1988 presented in S89, while filled triangles refer to points later than 1988. The smooth curve corresponds to a non-linear chisquare fit to the radius, for the energy conserving model of S89. The fit assumes there are no systematic differences in the estimates of the radius. The dotted curve represents the fit obtained using the parameters estimated by S89.

for the period 1901-1988 are obtained from S89, at 1990 from Anupama \& Prabhu (1993) and at 1993 from Slavin et al (1995). From the plot, the deceleration of the shell is evident, especially after 1950. There is also an indication that the deceleration rate may have decreased since 1987. This apparent change in the deceleration rate could be a result of systematic differences in the estimation of the radius of the shell by various authors, e.g. due to different procedures adopted, seeing and sensitivity. On the other hand, the observed change in the deceleration rate could be a true change, caused by density inhomogeneities in the ambient medium. Observations at future epochs are required to resolve this issue.

A proper motion study of the knots by Anupama \& Prabhu (1993) indicated the ambient density at the radius corresponding to the shell radius in 1990 to be lower than that estimated 

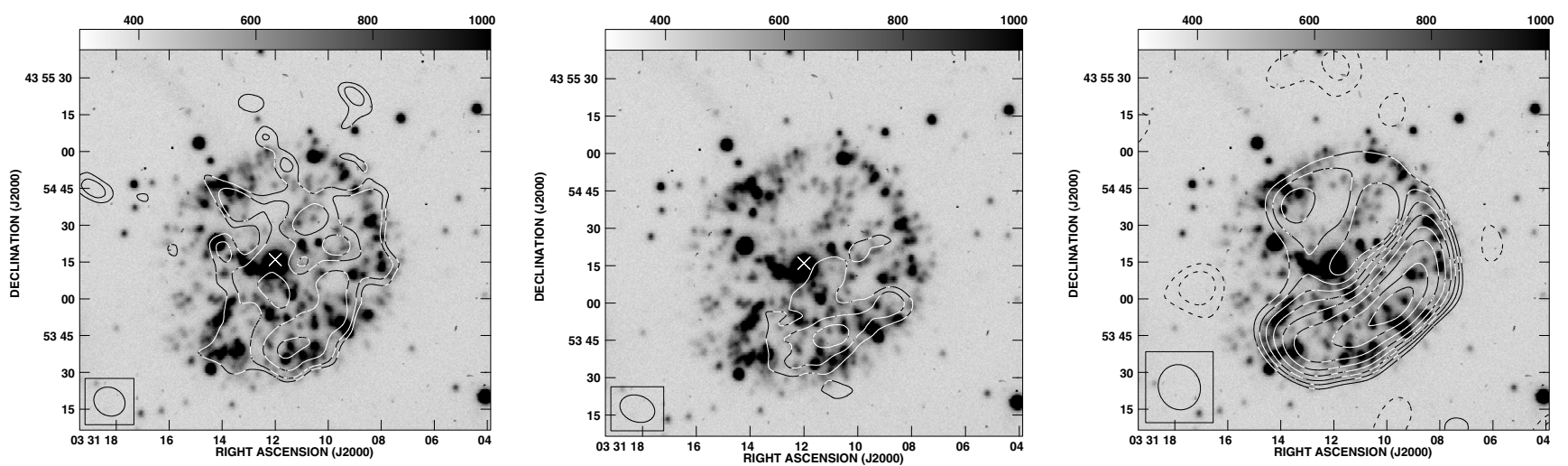

Fig. 6. $0.33 \mathrm{GHz}$ (left), $0.61 \mathrm{GHz}$ (center) and $1.4 \mathrm{GHz}$ (right) radio emission contours superimposed on greyscale image of the remnant in $\mathrm{H} \alpha+[\mathrm{NII}]$. The $1.4 \mathrm{GHz}$ data is from the VLA Data Archives.

at the location corresponding to the shell radius in 1987 by S89. Assuming the radius estimates are not affected by systematic differences, we fit both the energy conserving and momentum conserving shock interaction models of S89 to the evolution of the radius in the southwest deceleration zone. Using a non-linear chi-square fit (Levenberg-Marquardt algorithm: Press et al. 1993) for the expressions given in equations 9 and 10 in $\mathrm{S} 89$, we estimate an initial expansion velocity $\left(V_{0}\right)$ of $1240 \mathrm{~km} \mathrm{~s}^{-1}$ for the shell and the density of the ambient medium at $r=3.40 \times 10^{17} \mathrm{~cm}$, the current radius, to be $0.12 \mathrm{~cm}^{-3}$. This value is nearly seven times lower than the estimate of S89 and could explain the possible decrease in the deceleration rate. Density inhomogenities are clearly seen in the faint bipolar nebula associated with GK Per and it is likely that the current deceleration rate could change in the future as the shell encounters a change in the density of the ambient medium. The value of $V_{0}$ estimated here is lower than the value of $1700 \mathrm{~km} \mathrm{~s}^{-1}$ estimated by S89, but similar to the expansion velocities detected during the outburst in 1901. The estimated fit to the radius for the energy conserving model is also plotted in Fig. 5.

The model presented by S89 did not account for the asymmetry between the northeast and the southwest quadrants observed at that time. S89 note that there is no comparable interaction zone detected in the radio to the northeast and also a lack of bright knots in that region. It is interesting to note the increase in the number of knots in [NII] compared to the 1990 images, and the distinct flattening of the shell in the northeast region. Further, emission is detected from this region, although at low flux levels, in the radio also. Figure 6 shows the superposition of the $0.33 \mathrm{GHz}, 0.61 \mathrm{GHz}$ and $1.4 \mathrm{GHz}$ radio emission contours on the $\mathrm{H} \alpha+[\mathrm{NII}]$ image. From the figure it is seen that the radio emission at $0.33 \mathrm{GHz}$ and $1.4 \mathrm{GHz}$ is coincident with some of the brightest optical knots in the northeast quadrant. This is clear evidence of interaction of the shell with the ambient medium in that direction (see also Lawrence et al. 1995; Slavin et al. 1995). It appears that the interaction in the northeast direction is a recent phenomenon caused by the nova shell encountering a density enhancement in the ambient medium, and it would be interesting to study its future development in both the optical and radio.

\subsection{Evolution of the radio remnant}

The previous study of the nova remnant of GK Per in the radio by $\mathrm{S} 89$ indicated a global spectral index of -0.7 between $1.49 \mathrm{GHz}$ and $4.86 \mathrm{GHz}$, and that the spectrum turned over near $1 \mathrm{GHz}$. Based on this, S89 concluded that the nova remnant is somewhat different from supernova remnants, where the turnover occurs at much lower frequencies. The data presented here, on the other hand, indicate that the radio spectrum of the nova remnant follows a power law with a spectral index $\alpha \sim-0.85$ between $0.33 \mathrm{GHz}$ and $1.28 \mathrm{GHz}$ (Fig. 7). Unlike $\mathrm{S} 89$, we do not see a turnover around $1 \mathrm{GHz}$, and the radio spectrum of the GK Per shell closely resembles that of a supernova remnant.

We record a reduced flux density at $1.28 \mathrm{GHz}$ as compared to $\mathrm{S} 89$ by almost a factor of two. We do not detect the low brightness plateau around the ridge of emission that S89 had reported. It is likely that the nova shell has evolved over two decades. While the reduction in the flux density in the $1.28 \mathrm{GHz}$ could probably be explained by synchrotron ageing, one needs to explain the absence of a turnover in the present data. It is of interest to note that the flux density near $0.61 \mathrm{GHz}$ estimated by S89 agrees with our estimate within errors (see Table 3). Also, the spectral index between $0.61 \mathrm{GHz}$ and $0.408 \mathrm{GHz}$ is -0.85 (see Fig. 7) similar to the spectral index estimated for the present data. The flux density near $0.33 \mathrm{GHz}$ is however discrepant; we estimate a higher flux density compared to S89. It should also be noted that all the low frequency $(<0.5 \mathrm{GHz})$ data points in $\mathrm{S} 89$ have large error bars. If we assume that the flux density estimate at $0.33 \mathrm{GHz}$ by $\mathrm{S} 89$ to be in error and more likely similar to the present estimate, this would imply a steeper spectrum at the lower frequencies which has not changed in two decades. Alternatively, it is possible that the flux at $0.33 \mathrm{GHz}$ has indeed increased.

The evolution of the radio shell since the work of S89 is traced. The flux densities at $1.4 \mathrm{GHz}$ and $4.9 \mathrm{GHz}$ in $1997 \mathrm{in}-$ dicate that the spectral index between $1 \mathrm{GHz}$ and $5 \mathrm{GHz}$ has remained the same since 1984 (S89), at -0.7 , while the flux densities have decreased (see Fig. 7). The flux densities at these frequencies, together with the global spectral index at 1984 and 1997 are listed in Table 5. The flux densities imply a secular decrease of $2.1 \%$ per year. Assuming a similar secular decrease, 
Table 5. Flux density of GK Per around $1.4 \mathrm{GHz}$ and $4.9 \mathrm{GHz}$.

\begin{tabular}{ccc}
\hline \hline $\begin{array}{c}\text { Frequency } \\
\text { GHz }\end{array}$ & $\begin{array}{c}\text { Flux density } \\
\text { mJy }\end{array}$ & Spectral index $\alpha$ \\
\hline & S 89 & \\
1.490 & $20.6 \pm 1.6$ & -0.69 \\
4.860 & $8.7 \pm 0.5$ & \\
& 1997 & \\
1.425 & $14.9 \pm 0.4$ & -0.66 \\
4.900 & $6.6 \pm 0.2$ & \\
\hline
\end{tabular}

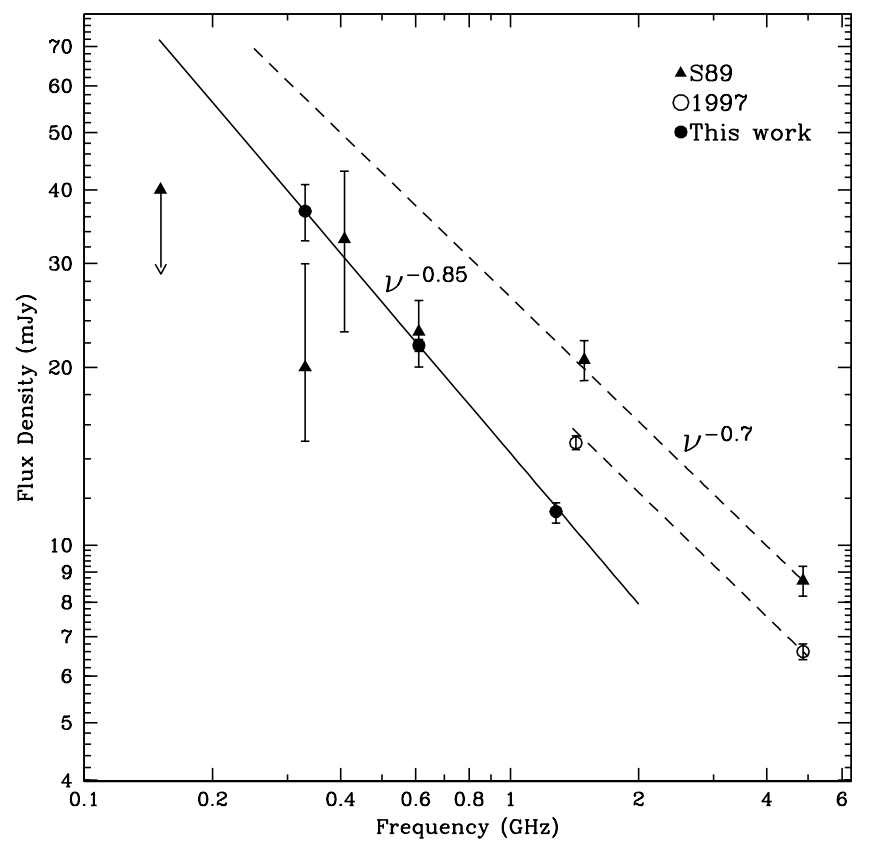

Fig. 7. Radio spectrum of GK Per. Filled circles correspond to the data presented here, filled triangles correspond to the data presented in S89, and open circles correspond to the flux densites in 1997. Also shown is the power law $v^{-0.85}$ for the spectrum between 330 and $1.28 \mathrm{GHz}$ (continuous line), and the power law $v^{-0.7}$ estimated by S 89 for the spectrum between $1.4 \mathrm{GHz}$ and $5 \mathrm{GHz}$ (dashed lines).

one would expect the flux density at $1.4 \mathrm{GHz}$ to be $13.1 \mathrm{mJy}$ in 2003, while the observed flux density at $1.28 \mathrm{GHz}$ is $11.4 \mathrm{mJy}$. It should be borne in mind that the presence of the three strong sources in the field in the $1.28 \mathrm{GHz}$ data could affect the flux density estimate to some extent. On the other hand, the secular decrease in the flux density could be different from the previous secular decrease of $2.1 \%$ per year, but we are unable to ascertain this due to the absence of data at higher frequencies for the same epoch. If one assumes the spectral index remains unchanged, then, based on the observed $1.28 \mathrm{GHz}$ flux density, we expect the flux density at $5 \mathrm{GHz}$ to be $4.6 \mathrm{mJy}$ in epoch 2003.

We discuss below the most interesting aspects of the evolution of the radio emission.

1. The spectrum of electrons above $1.4 \mathrm{GHz}$ follows a power law with an index -0.7 and below $1.4 \mathrm{GHz}$ follows a power law with index -0.85 . There is a break near $1.4 \mathrm{GHz}$, but the change in spectral index is negative and of a small magnitude $(0.15)$ contrary to what $S 89$ had concluded. This we believe could be due to the presence of at least two populations of electrons dominating the global emission at different frequencies. This is somewhat evident in Fig. 2. The radio continuum emission at $0.33 \mathrm{GHz}$ shows two peaks the strongest is distinct from the peak of emission near 1.4 GHz. The changes in the relativistic particle population could be caused by density changes in the circumstellar medium with which the nova shell interacts. Spectral index variations have been studied in detail in Cas A where different knots have been found to show different spectral indices (Anderson et al. 1991; Wright et al. 1999) and attributed to differences in the local particle acceleration conditions. It would be interesting to carry out a similar study of GK Per and study the spectral index variation across the remnant.

2. The nova remnant is experiencing a secular decrease in the flux density of about $2.1 \%$ per year at frequencies above $1.4 \mathrm{GHz}$ which has left the spectral index unchanged. Although the rate of secular decrease is higher in GK Per, we note that an annual secular decrease of about $1 \%$ has been well-studied in Cas A, which also shows a frequency dependent decrease (e.g. Shklovskii 1960; Baars et al. 1977; Agafonov 1996; Reichart \& Stephens 2000).

The secular decrease in the flux density in GK Per could be a result of adiabatic expansion of the remnant into the surrounding medium. The evolution of the optical shell indicates the shell could be in the energy conserving (adiabatic) phase. In this case, the flux density would vary as $S_{v} \propto$ $r^{-2(2 \alpha+1)}$, where $r$ is the radius of the shell and $\alpha$ is the spectral index (Shklovskii 1960). The ratio of the flux densities at two different epochs would be $S_{2} / S_{1}=\left(r_{2} / r_{1}\right)^{-2(2 \alpha+1)}$. Using the radii estimates at 1984 and 1997 from Fig. 5, the expected ratio of the flux densities is 0.53 . The observed ratios are $0.72(1.4 \mathrm{GHz})$ and $0.79(4.9 \mathrm{GHz})$, which are similar to the expected ratio (within errors), indicating that the secular decrease can be attributed to adiabatic expansion.

3. We estimate a higher $0.33 \mathrm{GHz}$ flux density compared to S89 (see Table 3). If this is indeed a true rise, then GK Per appears increasingly similar to Cas A which has shown anomalous increase in the flux density at $0.038 \mathrm{GHz}$ (Erickson \& Perley 1975; Read 1977a,b; Walczowski \& Smith 1985). The increase in the flux density in Cas A is explained by Chevalier et al. (1978) as being due to a fresh injection of relativistic electrons due to density variations in the vicinity of Cas A.

It would be interesting to model the emission from the nova remnant and arrive at a consistent model. However, before that, it is necessary to confirm (a) the secular decrease in GK Per, and (b) the increase in flux density at the $0.33 \mathrm{GHz}$. Clearly, there is a need for more multifrequency, nearly simultaneous data to have a better understanding of the evolution and nature of the radio shell of nova GK Per.

\section{Conclusions}

We present in this paper the evolution of the nova remnant of GK Persei, in the optical and low-frequency radio regions, since the previous detailed work of S89. 
1. The shell is boxy, asymmetric and clumpy in nature. The dimensions of the shell in the optical emission lines of [NII], [OIII] and [OII] are $108 \times 94 \operatorname{arcsec}^{2}, 104 \times 94 \operatorname{arcsec}^{2}$ and $99 \times 87 \operatorname{arcsec}^{2}$, respectively. The shell has expanded since the previous estimate in 1993 by Slavin et al. (1995). The evolution of the southwest interaction zone of the shell since 1916 indicates that the shell is decelerating due to shock interaction of the nova ejecta with its ambient medium (S89). Application of a simple model for the shock and its evolution to determine the time dependence of the radius of the shell in the southwest quadrant (S89) indicates that the shell is now expanding into ambient medium that has a density of $0.12 \mathrm{~cm}^{-3}$, compared to the ambient medium density of $0.8 \mathrm{~cm}^{-3}$ ahead of the shock in 1987 .

2. There are indications of a recent interaction of the nova remnant with the ambient medium in the northeast quadrant also. There is a distinct flattening of the shell, as well as an increase in the number and brightness of the knots in the region. The brightest optical knots in this region are also detected in the radio images.

3. The spectrum of electrons above $1.4 \mathrm{GHz}$ follows a power law with an index -0.7 and below $1.4 \mathrm{GHz}$ follows a power law with index -0.85 . This could be due to the presence of at least two populations of electrons dominating the global emission at different frequencies.

The flux densities at $1.4 \mathrm{GHz}$ and $4.9 \mathrm{GHz}$ estimated by S89 indicate a spectral index of -0.7 over that frequency range. The flux densities of the shell at the same frequencies in 1997 indicate no change in the spectral index, while the flux densities have decreased, indicating a secular decrease of $2.1 \%$ per year. We are unable to comment on the present evolution of the nova remnant at these frequencies due to a lack of observations.

The flux density that is estimated here at $0.33 \mathrm{GHz}$ is much higher compared to S89.

It is suggested that the observed evolution of the nova remnant in the radio is probably due to changes in the relativistic particle population caused by density changes in the ambient medium with which the shell interacts. The evolution of the GK Per remnant appears to be similar to that of young supernova remnants, in particular, the young supernova remnant Cas A.

4. The optical images also clearly show the extended bipolar nebula associated with mass loss processes that occurred during the evolution of the nova binary. This nebulosity has an hourglass shape that is flattened towards the southwest. The brightest regions of the nebula are the flattened portion in the southwest and a jet feature in the northeast. The [OIII] emission lies inwards of the $\mathrm{H} \alpha$ emission, except for the jet feature that is coincident in both emissions.

5. We also detect the extended HI bipolar emission feature associated with GK Per in our $21 \mathrm{~cm}$ images. The emission is detected at $4.9 \mathrm{~km} \mathrm{~s}^{-1}$, and extends over $\sim 15$ arcmin, which corresponds to a cloud of linear dimensions of 2.1 parsec at the distance of GK Per.
Acknowledgements. We thank the staff of IAO, Hanle and CREST, Hosakote, for their support during obervations. The facilities at IAO and CREST are operated by the Indian Institute of Astrophysics, Bangalore. We thank the staff of the GMRT who made these observations possible. GMRT is run by the National Centre for Radio Astrophysics of the Tata Institute of Fundamental Research. This work has made use of The NRAO Data Archives. The National Radio Astronomy Observatory is a facility of the National Science Foundation (USA) operated under cooperative agreement by Associated Universities, Inc. G.C.A. thanks K.E. Rangarajan for his help with the non-linear chi-square fit. Discussions with A. Mangalam and D. Bhattacharyya are acknowledged. We thank the referee for encouraging and very useful comments.

\section{References}

Agafonov, M. I. 1996, A\&A, 306, 578

Anderson, M. C., Rudnick, L., Leppik, P., et al. 1991, ApJ, 373, 146

Anupama, G. C., \& Prabhu, T. P. 1993, MNRAS, 263, 335

Baars, J. W. M., Genzel, R., Pauliny-Toth, I. I. K., \& Witzel, A. 1977, A\&A, 61, 99

Balman, S. 2002, in Classical Nova Explosions, ed. M. Hernanz, \& J. Jose, AIP Conf. Ser., 637, 365

Balman, S., \& Ögelman, H. B. 1999, ApJ, L111

Bianchini, A., \& Sabbadin, F. 1983, A\&A, 54, 393

Bode, M. F. 2004, in Asymmetric Planetary Nebulae III, ed. M. Meixner, J. Kastner, \& N. Soker, ASP Conf. Ser., in press

Bode, M. F., O'Brien, T. J., \& Simpson, M. 2004, ApJ, 600, L63

Bode, M. F., Seaquist, E. R., Frail, D. A., et al. 1987, Nature, 329, 519

Chevalier, R. A., Oegerle, W. R., \& Scott, J. S. 1978, ApJ, 222, 527

Couderc, P. 1939, Ann. Astrophys., 300, 788

Dougherty, S. M., Waters, L. B. F. M., Bode, M. F., et al. 1996, A\&A, 306, 547

Erickson, W. C., \& Perley, R. A. 1975, ApJ, 2000, L83

Lawrence, S. S., MacAlpine, G. M., Uomoto, A., et al. 1995, AJ, 109, 2635

McLaughlin, D. B. 1960, in Stars and Stellar Systems, ed. J. L. Greenstein (Chicago: University of Chicago Press), 6, 585

O'Sullivan, C., \& Green, D. A. 1999, MNRAS, 303, 5750

Perrine, C. D. 1902, ApJ, 16, 249

Press, W. H., Teukolsky, S. A., Vellerling, W. T., \& Flannery, B. P. 1993, Numerical Recipes (Cambridge: Cambridge University Press)

Read, P. L. 1977a, MNRAS, 178, 259

Read, P. L. 1977b, MNRAS, 181, 63

Reichart, D. E., \& Stephens, A. W. 2000, ApJ, 537, 904

Reynolds, S. P., \& Chevalier, R. A. 1984, ApJ, 281, L33

Ritchey, G. W. 1901, ApJ, 14, 167

Scott, A. D., Rawlings, J. M. C., \& Evans, A. 1994, MNRAS, 276, 353

Seaquist, E. R., Bode, M. F., Frail, D. A., et al. 1989, ApJ, 344, 805 (S89)

Shklovskii, I. S. 1960, SvA, 4, 243

Slavin, A. J., O’Brien, T. J., \& Dunlop, J. S. 1995, MNRAS, 276, 353

Tweedy, R. W. 1995, ApJ, 438, 917

Walczowski, L. T., \& Smith, K. L. 1985, MNRAS, 212, 27

Warner, B. 1995, Cataclysmic Variables (Cambridge: Cambridge Univ. Press)

Wright, M., Dickel, J., Koralesky, B., \& Rudnick, L. 1999, ApJ, 518, 284 